ÉGYPTE

monde arabe

\section{Égypte/Monde arabe}

9 | 1992

Vers une économie libérale?

\title{
Entreprises moyennes et risque égyptien
}

(étude de psychologie économique)

Jean-Claude Delaunay

\section{(2) OpenEdition}

\section{Journals}

Édition électronique

URL : https://journals.openedition.org/ema/1213

DOI : 10.4000/ema.1213

ISSN : 2090-7273

Éditeur

CEDEJ - Centre d'études et de documentation économiques juridiques et sociales

Édition imprimée

Date de publication : 31 mars 1992

Pagination : 47-66

ISSN : 1110-5097

Référence électronique

Jean-Claude Delaunay, « Entreprises moyennes et risque égyptien », Égypte/Monde arabe [En ligne], 9 | 1992, mis en ligne le 08 juillet 2008, consulté le 07 juillet 2022. URL : http://journals.openedition.org/ ema/1213; DOI : https://doi.org/10.4000/ema.1213

Ce document a été généré automatiquement le 7 juillet 2022

Tous droits réservés 


\section{Entreprises moyennes et risque égyptien}

(étude de psychologie économique)

Jean-Claude Delaunay

1 Au regard de ses dépenses de consommation et d'investissement, l'Égypte dispose de ressources réelles insuffisantes, celles-ci ne couvrant que $85 \%$ à $90 \%$ de sa demande ${ }^{1}$. L'Égypte vit aujourd'hui en important plus qu'elle n'exporte. Pour 1988/89, le déficit de sa balance commerciale est évalué à 7,5 milliards \$. Les raisons profondes de cette situation sont connues. À sa population, d'environ 56 millions d'habitants, s'ajoutent chaque année plus d'un million d'enfants, qu'il faut d'abord nourrir, puis éduquer, et auxquels il faudra ensuite procurer salaire et emploi. Chaque année, c'est désormais 450000 emplois agricoles et industriels qu'il conviendrait de créer. On comprend, dans ces conditions, la double pression, de consommation finale et d'investissement, qui s'exerce sur l'Égypte et la conduit à dépenser plus que ses moyens ne le lui permettraient ${ }^{2}$.

2 Jusqu'en 1985, cependant, cette économie a pu faire face tant bien que mal à ses obligations extérieures. Elle a pu, grâce aux recettes directes et indirectes tirées du pétrole, ainsi qu'à d'autres ressources en devises, régler sa dette annuelle et importer pour les ménages. Les services et les invisibles ont, en quelque sorte, équilibré le déficit de la balance commerciale. Il n'en a plus été de même après 1986. Ces recettes compensatrices diminuant, a-t-il semblé, de façon durable, l'Égypte s'est retrouvée devant l'alternative suivante : s'endetter très fortement ou changer de politique, sinon dans les objectifs, du moins dans les méthodes. Tel est le sens que l'on peut donner aux négociations engagées par l'Égypte avec le Fonds Monétaire International et la Banque Mondiale, ainsi qu'avec ses principaux créanciers, à partir de 1987. En 1991, il semble que l'Égypte se soit, plus résolument que par le passé, engagée dans la voie nouvelle que représenterait, pour elle, une économie de marché insérée dans le marché mondial. Pour atteindre les objectifs de croissance découlant de sa démographie, ce sont d'autres moyens qui seraient par elle mis en oeuvre, d'autres formes institutionnelles qui seraient par elle sollicitées. 
3 Le sujet abordé dans le présent article prend place dans l'ensemble des problèmes que l'on vient d'évoquer à grands traits. En effet, s'il est vrai que l'Égypte ne peut plus faire face au remboursement comme aux charges de son endettement, cela ne signifie pas, par autant, qu'elle doive se refuser de recourir à l'épargne internationale. Mais elle doit procéder à un certain nombre de changements, non seulement dans le volume de cette épargne, mais peut-être surtout dans le mode de son emploi. Ce qui peut signifier, audelà de l'instauration de nouveaux mécanismes économiques, le recours complémentaire à d'autres agents extérieurs que ceux auxquels elle était accoutumée. Précisément, il semble qu'aux grosses opérations commerciales de la décennie précédente, effectuées avec le secteur public égyptien par des entreprises de taille mondiale et financées par des prêts, doivent s'ajouter celles d'entreprises moyennes, venant produire en Égypte en y apportant leur savoir-faire et en engageant leurs capitaux dans le secteur privé de ce pays.

4 L'intérêt de cette solution est que, en contribuant à renforcer le tissu productif égyptien, elle favoriserait l'émergence ou la consolidation d'une catégorie locale d'entrepreneurs susceptibles d'impulser une dynamique améliorée de formation et de mobilisation de l'épargne intérieure. Comme le disait un banquier, début 1990, «ce n'est pas de prêts dont l'Égypte a besoin aujourd'hui, mais de sang nouveau ", entendant par là, nous semble-t-il, que le capital investi pour produire localement, avec le management adapté, est encore plus indispensable à ce pays, en raison de ses effets directs ou induits, que la vente de marchandises ou l'installation de gros équipements.

Une des questions qui se pose est alors celle de savoir si une solution de ce genre est possible et s'il existe des acteurs potentiels de sa réalisation. Pour chercher à y répondre, on est conduit à explorer des domaines moins précis que ceux de la macroéconomie ou de la modélisation économique, et pourtant tout aussi nécessaires à intégrer à la réflexion. La connaissance économique repose non seulement sur l'analyse des contraintes et des lois propres aux grandeurs caractéristiques de son champ d'observation, mais également sur l'analyse du comportement des acteurs de ce champ. Elle est aussi, d'une certaine manière, psychologie économique et étude des mentalités.

6 C'est avec cette préoccupation que l'on s'est rendu en Égypte, en mars 1990, pour tenter de cerner, à l'aide d'entretiens non directifs, l'ampleur et la nature du risque égyptien, tel qu'il est perçu par des entrepreneurs et des banquiers, français pour la plupart, mais travaillant presque tous dans ce pays depuis plusieurs années. La méthode utilisée a consisté à recueillir des opinions sur le sujet considéré3. C'est une limite évidente aux conclusions que l'on en a tirées. Cette méthode, cependant, a présenté un avantage. Elle a conduit à rassembler, en un temps très court, l'expérience de personnes directement impliquées dans la société égyptienne. Elle a donc permis, par observateur interposé, de construire un premier schéma de description et d'interprétation du problème étudié, que l'on peut définir comme étant celui d'une appréhension psychologique du risque égyptien et des conséquences que les agents économiques peuvent en tirer pour leurs décisions.

7 C'est pourquoi, avec les réserves que l'on vient d'énoncer, on montre que le risque encouru par une entreprise moyenne étrangère pour accéder au marché de l'Égypte est élevé mais que, cette étape une fois franchie, les perspectives de rentabilité y sont également fortes. Comment prendre place sur le marché égyptien sans commettre d'erreurs excessives, telle parait être la question majeure à laquelle doivent répondre 
des entreprises de taille moyenne pour s'y engager avec des chances raisonnables de succès.

8 Au terme de notre enquête, il apparaît que pour accéder au marché égyptien, un entrepreneur, surtout s'il ne dispose que d'une assiette financière réduite, se doit de traiter et d'appréhender avec le plus grand soin les trois problèmes suivants :

1. La délimitation de son propre marché;

2. La connaissance des lois et règlements du pays ainsi que leur mise en oeuvre par l'administration égyptienne ;

3. Le contexte psycho-sociologique de l'Égypte.

9 Une information insuffisante sur chacun de ces points pourrait le conduire à une décision erronée, aux conséquences très coûteuses pour son entreprise. Pour ce qui concerne la connaissance de l'administration ou du contexte culturel égyptiens, le savoir qu'il accumulera ne lui servira pas seulement au moment de sa décision initiale. Elle lui sera utile à tout moment de son action productive. L'Égypte est sans doute un marché. Mais c'est aussi, et peut-être surtout, un état d'esprit.

\section{La délimitation du marche propre à l'entreprise}

10 La prospection du marché pour lequel elle se propose de produire régulièrement est, pour une entreprise, une opération aussi normale que nécessaire. Les principales particularités égyptiennes sont, de ce point de vue, les suivantes.

\section{L'existence d'une grande disparité dans les revenus des populations}

11 En 1988, le revenu annuel moyen aurait été en Égypte d'environ 650 \$ (estimation de la Banque Mondiale). Mais il ne s'agissait que d'une moyenne arithmétique. D'après l'un de nos interlocuteurs, «au Caire, on se croirait dans un pays où les revenus par tête atteindraient 5000 \$. Mais le long du Nil, les fellahs vivent dans un monde à culture vivrière, avec peu de moyens ». Et au Caire même, ville riche et brillante, existent d'importantes zones de pauvreté. Ces disparités peuvent induire une perception inexacte des potentialités du marché égyptien et de la stratégie à suivre à son égard, tant en ce qui concerne le prix que la qualité des produits offerts par l'entreprise.

12 En s'en tenant aux seuls biens de consommation, on distinguera tout d'abord ceux que peuvent acheter les catégories sociales à faible revenu. Il s'agit de produits qualifiés de bas de gamme, pour lesquels le prix constitue la variable déterminante de leur vente.

13 S'engager dans leur production avec un objectif de qualité serait illusoire, dans la mesure où la concurrence des importations en provenance d'Asie du Sud-Est, pour des produits semblables, est extrêmement forte.

14 Il n'en est déjà plus de même pour ce qui concerne les catégories sociales moyennes, disposant d'un certain revenu et ayant des habitudes urbaines de consommation. Les prix sont, dans ce domaine de produits, un argument de concurrence évident. Mais une stratégie de qualité, adaptée au milieu, peut être développée, notamment pour combattre les imitations et labels frauduleux dont ces produits sont l'objet.

15 Enfin, pour les catégories sociales plus aisées, ainsi que pour les touristes d'affaire ou d'agrément, qu'ils viennent de pays arabes ou occidentaux, la qualité et l'entretien, 
quand il s'agit de produits durables, sont alors des variables concurrentielles de premier plan.

16 L'argument de qualité, souvent évoqué par les entrepreneurs français pour justifier les prix élevés qu'ils pratiquent, ne peut donc être utilisé sans discernement dans un pays où les revenus sont très différenciés et où la concurrence est forte, pour toutes les gammes de produits. Quand il est pertinent, cet argument doit être adapté au milieu et non pas envisagé sous un angle étroitement technique. On notera enfin que, ici comme ailleurs, il ne peut concerner uniquement le produit stricto sensu, mais qu'il doit aussi s'appliquer à son usage dans le temps (entretien et réparation). La rapidité d'approvisionnement du marché en pièces détachées, àdes prix tenant compte de la concurrence, apparaît alors comme une préoccupation indispensable.

\section{Des secteurs productifs déjà occupés}

17 L'Égypte est un pays déjà industrialisé. Beaucoup plus que le Yémen, et plus que l'Algérie. Un entrepreneur étranger qui en explore le marché s'y heurte non seulement à la concurrence commerciale d'autres entreprises étrangères mais encore à celle, directement productive, d'entreprises locales. Un homme d'affaires égyptien donne l'exemple d'une petite société française qu'il avait contactée, et qui fait des pièces de rechange pour toutes sortes d'appareils: «Elle a essayé de travailler ici. Mais les Égyptiens peuvent faire la même chose et moins cher. Il faut trouver le créneau.» L'entrepreneur doit donc cerner son marché potentiel et en évaluer l'ampleur, pour éviter d'y investir de manière excessive.

Il peut avoir le réflexe de recourir aux services d'une société de marketing. Mais il court alors un autre risque, résultant de ce que la qualité de l'offre de prestations en ce domaine n'est peut-être pas encore très assurée. De façon brutale, un manager français ayant été confronté à ce problème au début des années quatre-vingt, énonce: "Les conseils en marketing, ici, ça ne vaut rien. Les études de marché, il faut les faire soimême... La publicité, ça ne vaut rien non plus, parce qu'il n'y a pas de panel... Il faut se débrouiller tout seul, recouper les informations, se constituer une base de données. Rien d'équivalent à Nilsen, ici. $»^{4}$ On doit noter cependant, pour contrebalancer l'opinion très négative de cet entrepreneur, l'existence, sur la place, de quelques sociétés, telle « Americana » (publicité et marketing), ayant une bonne réputation.

Quoiqu'il en soit, pour obtenir des informations approximativement fiables, l'entrepreneur en question risque d'avoir à se tourner vers des sources moins spécialisées que ne le seraient des cabinets de marketing européens. Il lui appartiendra alors de faire la synthèse intelligente des informations qu'il aura recueillies. Les banques apparaissent comme étant des lieux indiqués pour l'obtention d'un savoir concret sur le marché égyptien, sur ses acteurs, ses évolutions. À cela s'ajoute, en Égypte, l'existence d'un appareil statistique public de qualité relativement bonne comparativement à celles d'autres pays du Moyen-Orient, même si elle est très discutable au plan de ses résultats macro-économiques. Pour des raisons administratives, certains domaines - celui de la pharmacie, par exemple - peuvent être mieux explorés que d'autres. En outre, il existe des bottins, des annuaires et plusieurs millions d'Égyptiens sont abonnés au téléphone. Enfin, une autre source possible d'information est celle que constituent les administrations économiques et chambres de commerce accompagnant généralement les ambassades. 
On note sur ce point des différences d'appréciation dans les entretiens. La Chambre de commerce allemande, au Caire, est perçue par exemple comme étant plus apte à satisfaire ce besoin que d'autres organismes. D'après un entrepreneur français, "les Allemands en savent beaucoup plus que d'autres... Ils sont davantage capables de répondre à une question précise. » De fait, il existe, au sein de la Chambre germanoarabe du Caire, un département de marketing, et de jeunes stagiaires de facultés économiques ou d'écoles de commerce peuvent être impliqués dans ce travail. De son côté, la chambre américaine de commerce en Égypte (Amcham) entretient vraisemblablement des relations professionnelles avec les professeurs du département de management de l'Université américaine du Caire. Elle peut, également, sous-traiter des études à des cabinets américains ou utiliser d'autres voies, eu égard au poids économique des États-Unis dans le pays. Cela dit, étant donné tout un ensemble de difficultés techniques et linguistiques, l'évaluation par un entrepreneur, de son marché potentiel, ne sera, dans le meilleur des cas, qu'un ordre de grandeur, une approximation. Le Poste d'expansion économique (Ambassade de France), dans le cadre renouvelé de son travail depuis l'adoption de la Charte nationale de l'exportation, fait des efforts en ce sens pour le compte d'entreprises françaises ${ }^{5}$.

\section{L'administration égyptienne}

21 Le risque représenté par l'administration égyptienne, avec lequel un entrepreneur étranger sera nécessairement en rapport pour sa décision d'investissement, peut être appréhendé sous deux angles. certains membres de son personnel. Bien que leur mention puisse sembler désobligeante, le fait que les élites égyptiennes, ou bien d'autres personnes en Égypte s'en désolent, autorise à en faire état sans détour. On n'y insistera pas davantage. Il s'agit ensuite, et peut-être surtout, du comportement de certains responsables administratifs égyptiens pour lesquels il semble qu'il soit difficile de prendre des décisions et de s'y tenir, indépendamment de toute question de rigueur morale ou professionnelle.

25 L'absence totale de délégation de pouvoir qui prévaut dans cette administration aboutit à ce qu'il revient aux supérieurs hiérarchiques de prendre toutes les décisions. Les formulaires et dossiers s'entasseraient sur leurs bureaux et resteraient très longtemps sans réponse.

Selon un avocat, l'autorisation pour une société étrangère d'exercer son activité en Égypte « s'obtient au bout de 18 mois ou 2 ans, alors qu'existent des dispositions légales selon lesquelles cette autorisation peut être automatiquement acquise au terme de 60 
jours, à défaut de réponse de la part de l'administration ». Mais, ajoute-t-il, « en Égypte, ça ne se passe pas comme ça ».

L'autre risque administratif est, pour un entrepreneur, celui découlant des pratiques relatives à la loi et à la réglementation égyptiennes.

Notons, tout d'abord, le caractère inconfortable, pour un investisseur étranger, de la loi sur les investissements (loi $230 \mathrm{du} 20$ juillet 1989), prise en remplacement de la loi $43 \mathrm{du}$ 19 juin 1974, elle-même amendée en $1977^{6}$. L'interprétation généralement donnée de cette législation est que, bien que témoignant d'une évolution favorable aux apporteurs de capitaux, décidés à travailler durablement et productivement en Égypte, elle se trouve en continuité avec les restrictions de la précédente. Selon un banquier, « on ne sait toujours pas si le pays est libéral ou socialiste. Ces deux systèmes sont mélangés et ça n'est pas favorable à l'économie dans son ensemble. " Dès lors, la tentation est grande, pour un entrepreneur, de se tourner vers des pays dont la législation est beaucoup plus claire de ce point de vue, qu'il s'agisse de ceux du Maghreb ou de la Turquie.

Mais en supposant qu'il accepte ce risque global, il semble qu'il doive anticiper diverses sortes de difficultés législatives ou réglementaires.

La première concerne l'extrême lenteur de parution de certains décrets d'application de lois déjà votées, ou, au contraire, la grande vitesse de leur changement. C'est ainsi, par exemple, que la réglementation fiscale changerait très rapidement. Un entrepreneur français fait ainsi état d'une expérience personnelle. «J'avais commandé un véhicule et il y avait un certain droit de douane à ce moment-là. Mais quand j'ai reçu le véhicule, le taux avait changé et j'ai payé $70 \%$ de taxes en plus. Dans ce pays, il y a des pièges. En particulier celui de la grande mobilité des droits de douanes. »

31 La seconde difficulté concernerait la possibilité, pour une entreprise, d'importer des matières premières sans coût excessif. Comme il est compréhensible, l'État égyptien favorise l'intégration des nouveaux investisseurs et des producteurs locaux, pour réduire les importations. Encore faut-il que les produits locaux conviennent. Un industriel français implanté en Égypte, déclare sur ce point que son entreprise «importe le PVC dont elle a besoin. On en fabrique en Égypte, mais il est de très mauvaise qualité. Nous avons mentionné dans notre contrat d'implantation que tel type de PVC serait importé. L'important étant de prendre cette précaution au départ, pour se couvrir. $»^{7}$

32 Un entrepreneur étranger a donc tout intérêt à prospecter avec soin le champ de ses futurs fournisseurs égyptiens, pour apprécier précisément la qualité de leurs produits. Il a, en outre, intérêt à se réserver la possibilité contractuelle d'importer certains des composants dont il aura besoin. La nouvelle législation concernant les importations et visant à leur libéralisation (juin 1991) pourrait, il est vrai, modifier cet aspect de son travail.

33 Là troisième difficulté se rapporte à la politique d'exportation. En effet, une entreprise s'installant en Égypte peut envisager d'exporter une partie de sa production. Cette orientation serait conforme à celle du gouvernement égyptien. Or voici ce que dit l'un de nos interlocuteurs : «On parle beaucoup, ici, de l'exportation. Mais les problèmes soulevés par l'administration égyptienne sont énormes. Une entreprise française qui produit en Égypte et qui exporte une partie de sa production perd un temps considérable en formalités de toutes sortes. En effet, normalement, le produit 
comprend des composants importés et ceux-ci ne sont pas taxables à l'exportation. Mais le règlement veut que ces composants importés soient vus montés sur le produit exporté. Or ça n'est pas évident. La fabrication peut donc prendre quinze jours. Puis il y a trois ou quatre mois d'attente pour l'exportation." Sur ce point encore, la libéralisation du régime des importations pourrait simplifier les procédures de la réexportation.

\section{Le contexte psychologique et social}

Une entreprise moyenne, dont l'expérience internationale aurait principalement porté, jusqu'alors, sur des pays industrialisés, se doit de prendre la mesure des différences existant entre ces pays et l'Égypte.

5 Certes, l'Égypte est proche de l'Europe, comme de la France, tant au plan affectif que géographique. Mais en même temps, il est vrai, le contexte anthropologique en est différent. Une décision d'investissement dans ce pays serait naïve si elle reposait sur la conviction que les méthodes apprises en d'autres lieux y sont aisément transposables.

6 En s'efforçant d'aller au-delà des apparences («Les entreprises en Égypte, dit un entrepreneur français, s'il y en a de très performantes, font parfois penser à la "Cour des miracles" »), les principales différences entre l'Europe et l'Égypte doivent être trouvées dans les notions qui structurent le plus essentiellement les comportements, à savoir celles de l'espace et du temps. La mondialisation contemporaine des économies conduit les chercheurs à s'intéresser activement au décodage de ces écarts 8 . Mais, pour l'instant, on doit surtout se satisfaire d'en reconnaître l'existence et d'adopter, à leur égard, une attitude expérimentale et pratique. Ces différences ont une incidence économique forte. Pour ne pas en rester au constat, banal quoique nécessaire, de la très grande importance des contacts personnels, on fera état ci-après de trois remarques apparues dans les entretiens.

7 Les deux premières ont surtout trait aux problèmes que risque de rencontrer un entrepreneur étranger, dans l'hypothèse d'une association avec un partenaire local.

En effet, le temps figurant dans la « mens » égyptienne semble être un temps immédiat. De là résulte, au plan économique, qu'une opération de prévision, de planification, de budgétisation sera plutôt conçue, par un associé égyptien, comme un exercice formel. D'après un entrepreneur, "un plan est effectué principalement pour faire plaisir ». Mais celui-ci n'aura pas de suite. Pour un autre interlocuteur, «dans les affaires, on butte tout le temps sur ce problème du temps. Une chose doit être faite dans un certain temps. Mais elle prend plus de temps que prévu sans que cela gêne celui qui est en retard... On pourrait penser que le contact avec le monde occidental aurait pu changer quelque chose à ce comportement. C'est beaucoup plus difficile.»

Une autre remarque ressortant des entretiens consiste à noter la tendance qu'auraient les managers égyptiens à installer des capacités de production excessives par rapport aux possibilités du marché. Un entrepreneur travaillant dans une entreprise conjointe explique que ses partenaires « ne conçoivent pas les problèmes de la même façon que la partie française. Ils veulent en quelque sorte la plus belle usine. Ils souhaiteraient que l'on doublât la chaîne. Car, disent-ils, si celle qui est installée tombe en panne, cela entraînera une perte d'argent. C'est ainsi qu'avant notre association, ils avaient acheté une machine à empaqueter couvrant dix fois le marché égyptien. » Le temps de ces entrepreneurs comme temps immédiat les conduit donc, selon cet interlocuteur, à 
vouloir faire face aux événements qui se produisent sans lier la dépense présente aux rendements futurs actualisés, ce qui est l'ambition du calcul économique. On pourrait, dans le même sens, noter l'absence quasi générale des pratiques préventives dans la surveillance et l'entretien du matériel.

La troisième remarque recueillie dans les entretiens à propos du temps concerne cette fois les travailleurs égyptiens et plus particulièrement leur productivité. Il est clair que la productivité du travail en Égypte est inférieure au niveau européen du secteur correspondant. La différence des équipements, de l'organisation des tâches comme des salaires, explique sans doute une partie de cette différence des résultats. Mais le comportement du facteur travail, sa façon de se situer dans le temps et par rapport à lui, apparaît également important à prendre en compte.

41 Ce constat étant fait, et d'un point de vue pratique, la question que peut se poser un entrepreneur envisageant d'investir en Égypte est celle de savoir comment accroître, s'il est possible, la productivité du travail de ses futurs salariés. Sera-t-il aisé, pour lui, de modifier, même avec des salaires très sensiblement plus élevés que la moyenne des salaires égyptiens, un comportement dont les fondements constituent la personnalité de base de ceux qui travailleront dans son entreprise? Les entretiens permettent de dégager, sur ce point, deux axes de réflexion.

42 Le premier est qu'il ne doit pas s'attendre à une modification radicale et instantanée des comportements au travail. L'apprentissage de la productivité du travail sera très vraisemblablement, dans son entreprise comme dans celles l'ayant précédée, le résultat d'une longue patience, susceptible de prendre plusieurs années d'un effort continu. Investir en Égypte, c'est s'atteler à une tâche durable de cinq à dix ans, avant d'en récolter les bénéfices.

Le second axe de réflexion est que la mise en rapport directe du travailleur et de son temps de travail suppose l'existence d'étapes intermédiaires, simples à comprendre. En effet, la productivité est un rapport numérique entre une quantité de produits et une quantité de temps. Or le temps, comme on vient de l'indiquer, est pour un Égyptien et dans la profondeur de sa personnalité, tout à fait différent du temps occidental. En revanche le produit, en tant que résultat du travail, fait partie de l'expérience sensible des uns et des autres.

En sorte que la quantité, et surtout la qualité de la production, apparaissent comme étant les leviers prioritaires d'une phase initiale, éventuellement longue, d'amélioration de la productivité du travail d'une telle entreprise, la formation et la rémunération étant les compléments nécessaires de cette façon de procéder. C'est sur cette base concrète, faisant intervenir les notions de soin professionnel, d'hygiène, de propreté de l'entreprise, de sécurité des opérations, de surveillance du matériel que peut s'édifier, à l'aide de solutions spécifiques, l'apprentissage de la maîtrise directe du temps par ceux qui travaillent.

Pour résumer cette première partie, on dira donc que l'enquête à laquelle il a été procédé a rendu sensible l'existence, pour une entreprise étrangère moyenne susceptible d'investir durablement en Égypte, de trois difficultés majeures. Elles sont relatives :

1. à une perception erronée du marché potentiel et des équipements nécessaires ;

2. à une connaissance insuffisante du comportement de l'administration égyptienne et de ses règles ; 
3. à une appréciation superficielle des structures fondamentales de la personnalité égyptienne, telle qu'elle s'exprime dans sa compréhension du temps et de son rapport à lui.

Ces difficultés entraînent la possibilité de risques élevés. En effet, un tel entrepreneur a peu de chance d'être, spontanément et simultanément, anthropologue, juriste, statisticien, ergonome, en plus de sa propre compétence technique et managériale. S'il connaît ses limites et n'hésite pas ordinairement à recourir à des conseils externes, il devra le faire a fortiori en Égypte. Le banquier et le juriste lui seront indispensables, et il trouvera dans ce pays, pour ces deux secteurs, les supports les plus convenables. En revanche, pour ce qui concerne l'exploration du marché ou la mise en oeuvre d'une politique créatrice de gestion de sa main d'oeuvre, et de rapport avec ses fournisseurs, il sera beaucoup plus livré à lui-même. Il devra donc prendre une décision difficile et de conséquence immédiate, avec une perspective éloignée de rentabilité. C'est pourquoi, sans doute, l'un de nos interlocuteurs désigne une opération de ce genre comme étant une « aventure ». Un autre mentionne à ce propos, le « besoin d'un esprit pionnier »".

Cependant, si le risque d'accès au marché égyptien est élevé, il ne l'est pas plus que dans bien d'autres marchés du monde. En outre, les perspectives de rentabilité en Égypte, même inscrites dans une démarche de moyen et long terme, y sont également élevées. Quels sont donc ces avantages compensant les inconvénients que l'on vient d'évoquer?

Accéder au marché égyptien par le biais d'une entreprise, en comprendre le contexte administratif et humain, trouver des partenaires ou des fournisseurs fiables, gérer la main-d'oeuvre, sont des opérations délicates. Une expression revient souvent dans les entretiens pour les caractériser, à savoir que l'Égypte serait un pays " plein de pièges ».

Mais en même temps, les possibilités en ont été soulignées de façon convergente. Tout d'abord l'Égypte, en raison de sa masse démographique et de sa position géographique, représenterait un potentiel important de production et de vente. Ensuite, ce pays dispose d'une main-d'oeuvre qualifiée, tant dans les domaines intellectuels que manuels. Les coûts salariaux y sont peu élevés. Complémentairement, les techniques adaptées au système productif égyptien font partie de celles qu'une entreprise européenne moyenne connaît et maîtrise sans difficulté.

50 De cela résulte que les profits que l'on peut escompter dans ce pays, si l'on a su s'y implanter, sont, à terme, élevés. Ce sont ces aspects que l'on se propose maintenant d'examiner.

\section{Un marché déjà important, au potentiel prometteur}

51 Bien que les personnes interrogées aient presque toutes insisté sur les "pièges" de l'Égypte, le caractère prometteur et déjà stimulant du marché de ce pays apparaît aussi nettement. "Le marché est énorme », dit un banquier. Pour un autre, "personne ne connaît l'Égypte, et il y a un manque de dynamisme à son propos. C'est un potentiel de production fabuleux, situé à quatre heures de l'Europe... C'est un capital de production et de consommation.» Ou bien encore, "malgré la situation actuelle, l'Égypte est un marché suffisamment vaste, où les besoins sont assez grands pour que des entreprises moyennes puissent y faire des affaires, même si on n'est plus dans les années quatrevingt.» Bien que se soient aussi exprimées des opinions plus réservées, il ressort 
globalement que le marché égyptien est apprécié positivement. En voici les principales raisons.

\section{Un marché offreur}

52 L'entreprise privée est, par définition, de la nature d'un pari. L'incertitude qui, au moment de son lancement, entoure son avenir peut être, cependant, plus ou moins grande. Or, l'une des caractéristiques du marché égyptien est que l'offre y est dominante. Le rapport à l'usage n'a pas encore atteint le degré de maturité qu'il connaît dans les pays occidentaux. Les besoins n'y sont pas saturés avec la même intensité. «Ici, dit l'un de nos interlocuteurs, le marché est un marché de fournisseurs ou de prestataires de services. " Pour un autre, «nous sommes dans un marché de producteurs. Tant que l'on fait un produit qui touche la 'middle-class', on est toujours pris. »

53 Malgré la contraction des affaires au cours des dernières années, malgré l'accentuation de ce mouvement avec la crise du Golfe et les mesures de libéralisation en cours d'application, il existe, en Égypte, un revenu produit intérieur. Au flux de dépenses qu'il induit s'ajoute celui engendré par les capitaux arabes et islamiques s'investissant dans ce pays. Lors de la onzième session de leur Conseil ( $(\mathrm{CG})$ à Doha, en décembre 1990, les pays du Golfe ont envisagé la réorientation de leurs flux financiers en fonction d'objectifs visant la stabilité régionale. L'Égypte pourrait être à terme, avec l'Iran, l'un des bénéficiaires importants de cette nouvelle politique. L'excellence des relations de ce pays avec la Libye, si elle se maintient, pourrait avoir le même effet.

\section{Un marché bien desservi}

Une autre caractéristique notable du marché égyptien, pour une entreprise, est qu'il est desservi par une infrastructure convenable, en matière de transports terrestres, maritimes, aériens, comme dans les domaines de la production d'énergie et des télécommunications. On compte, en Égypte, environ quatre millions d'abonnés au téléphone, dont $60 \%$ au Caire et $35 \%$ à Alexandrie. Le réseau bancaire y est dense et diversifié, les banques françaises étant surtout représentées par des entreprises conjointes à statut public égyptien, et par des banques «off shore» travaillant (mais jusqu'à quand, il est vrai, dans le contexte réglementaire actuel?) avec leurs propres ressources en devises. D'autres éléments, tels que des Universités encore prestigieuses, une presse vivante et diversifiée, un nombre important d'excellents juristes de droit international des affaires, contribuent à créer autour du marché un environnement favorable.

\section{Un marché bien situé}

Enfin, l'Égypte est perçue comme étant bien située dans l'ensemble régional araboafricain. Sans doute ce facteur géographique ne peut-il être encore pleinement exploité, en raison notamment de l'insuffisance des transports entre ce pays et ceux de la Péninsule arabique ou de l'Afrique proche. Mais, dans un calcul de long terme, il pourrait être pris en considération, d'après nos interlocuteurs, selon les trois axes suivants : 


\section{La Libye et le Soudan}

$$
\text { de l'Égypte. Ce n'est sans doute pas la plus développée aujourd'hui. Mais des }
$$
changements peuvent intervenir et en accroître l'intérêt.

\section{Les pays arabes du Moyen-Orient}

Cette zone possible d'extension des affaires est plus souvent citée dans les entretiens. Par exemple, dit un banquier, « à partir du Caire, on peut toucher tous les Arabes. Ils viennent ici en vacances. Inversement, les Égyptiens vont dans les autres pays du Moyen-Orient. L'Égypte est un peu le pivot de cet ensemble régional. » Ou bien encore, de la part d'un entrepreneur implanté en Égypte, « l'usine s'occupe de production et a une clientèle en Égypte. Nos autres clients seront ceux des pays du Moyen-Orient. Car l'implantation égyptienne offre des facilités. Cela paraît être une bonne plate-forme. » On peut faire, toutefois, la remarque suivante. Outre les transports ou la pratique de la législation, déjà mentionnés comme limites à cette politique régionale d'exportation, existe aussi le fait que les entreprises implantées en Égypte sont quelquefois représentées commercialement au Moyen-Orient. Leur installation en Égypte, avec une perspective d'exportation, suppose donc la réorganisation de leur réseau de distribution et de relations avec leurs anciens agents commerciaux. Mais ces modifications, notamment dans la Péninsule arabique, sont toujours fort complexes.

\section{Les pays d'Afrique}

Enfin, selon l'un de nos interlocuteurs, « aujourd'hui, l'Égypte regarde vers le Sud, pas seulement le Sud proche, mais vers des pays lointains. Peut-être n'ont-ils pas été encore capables de s'y implanter. Mais ils y pensent. Ils ont des relations avec le Sénégal, le Nigeria, l'Ouganda, le Kenya... Ils regardent avec un oeil attentif les sources du Nil.»

La géopolitique des affaires qui se développeront régionalement à partir de l'Égypte est sans doute difficile à anticiper et les opinions que l'on a évoquées ne sont pas des preuves du contour qui sera le sien. Cela dit, même pour une entreprise moyenne, il n'est pas interdit de raisonner à dix ou à vingt ans. Or dans l'immédiat, l'Égypte constitue une base de production. De plus, elle est dotée de zones franches avantageuses, non saturées et où la main-d'oeuvre est peu coûteuse. À plus long terme, on peut imaginer, comme cet autre entrepreneur, le scénario suivant : «L'Égypte étant africaine et l'Afrique étant un réservoir de matières premières, l'Égypte pourrait être un transformateur de ces matières premières, les pays arabes étant les bailleurs de fonds et les acheteurs de ces produits. L'Égypte a de l'eau, de bonnes conditions climatiques, des débouchés maritimes. » Il ne s'agit, bien sûr, pour l'instant que d'une hypothèse. Mais elle peut intervenir dans une décision de long terme.

\section{Les facteurs de production}

Ces caractéristiques générales favorables, relatives au marché égyptien, ayant été énoncées, on se propose maintenant d'examiner celles relatives aux facteurs de 
production. En distinguant capital technique et travail, les éléments de profitabilité pour chacun d'eux sont, à grands traits, les suivants ${ }^{10}$.

\section{Le capital technique}

61 Deux points ont été surtout soulignés dans les entretiens. Le premier concerne le niveau technique requis pour produire en Égypte, dans les branches où une entreprise étrangère pourrait investir. D'après l'un de nos interlocuteurs, ce niveau correspond à celui des années 1950-1960 en Europe, c'est-à-dire à un équipement mécanisé, mais non automatisé : «La haute technologie, ce serait, ici, une erreur. Il n'y a pas le personnel qualifié pour l'assurer. Il faut utiliser les outils de production des années 50/60. » Une telle position paraît conforme à la situation égyptienne : des équipements de haute technologie supposent de gros investissements, un personnel spécialement qualifié, accoutumé aux méthodes modernes de production, d'entretien et de vente. Or la maind'oeuvre égyptienne est en phase d'apprentissage des formes contemporaines de la productivité du travail et de ses compléments nécessaires. En outre, le marché égyptien n'est pas assez vaste, ou suffisamment ouvert sur d'autres marchés, pour absorber les quantités susceptibles d'être produites avec de tels équipements.

Une entreprise moyenne, visant à produire en Égypte, n'aura donc pas à se préoccuper de « veille technologique ». Les principaux problèmes qu'elle aura à résoudre, au plan des facteurs de production, seront, sur le fondement de techniques maitrisées, ceux de la délimitation de sa capacité de production et ceux de l'organisation du travail. Sans pouvoir se satisfaire du réemploi des rebuts techniques de ses entreprises européennes, il est assuré, compte tenu du caractère offreur du marché, de les utiliser sur toute leur durée de vie. "Il ne faut pas oublier qu'en Égypte, dit un banquier, on ne parle pas d'obsolescence, mais d'amortissement physique. Les machines travaillent jusqu'à ce qu'elles soient physiquement usées.»

\section{Le travail}

63 On distingue ici deux aspects du facteur travail: son coût et ses caractéristiques professionnelles.

\section{Le coût du travail}

Pour diverses raisons, et en particulier l'existence d'un système public de plus en plus fictif d'emploi garanti tirant l'ensemble des rémunérations vers le bas, il apparaît que le coût salarial de la main-d'oeuvre égyptienne est faible en comparaison des standards européens ou même d'autres pays arabes.

À titre d'illustration, voici deux niveaux salariaux mensuels et ponctuels relevés lors de notre enquête, l'un pour le secteur public égyptien, l'autre pour le secteur privé, à management européen. Ces relevés sont incomplets. Ils ne prennent peut-être pas les primes en compte. Ils ne sont pas datés avec précision et on ne les reproduit ici que comme des illustrations particulières. 


\begin{tabular}{|l|c|c|}
\hline \multicolumn{1}{|c|}{ Oualifications } & $\begin{array}{c}\text { Secleur public } \\
\text { (institul médical) }\end{array}$ & $\begin{array}{c}\text { Secteur privé } \\
\text { (produclion) }\end{array}$ \\
\hline $\begin{array}{l}\text { Directeur de département } \\
\text { Ingénieuŕ } \\
\text { Technicięn }\end{array}$ & 1145 (médecin) & $\begin{array}{c}1000-1500 \\
400-500 \\
250-300\end{array}$ \\
Mancuvre & 85 & 150 \\
Femme de ménage & & \\
\hline
\end{tabular}

Unité : livre égyptienne fois plus forte et plus hiérarchisée que dans le secteur public. Pour l'entreprise privée, le coût salarial mensuel d'un directeur de département situé en haut de l'échelle aurait été, en mars 1990, compris entre 1500 et $2200 \mathrm{FF}$, en fonction du taux de change de l'époque, et aurait correspondu à dix fois celui du manoeuvre. Même si l'on ne peut comparer directement les salaires de deux pays en les exprimant dans une monnaie commune, cette première approche montrerait que le SMIC mensuel net forfaitaire, en France (mars 1990), aurait été d'environ deux fois supérieur, avec ces données, au salaire correspondant d'un directeur égyptien de département. Outre cette différence entre les salaires pratiqués en France et en Égypte, l'avantage salarial, pour un entrepreneur s'implantant en Égypte, peut être appréhendé de deux manières complémentaires.

1 - Certes, les salaires que son entreprise aura à verser seront supérieurs à ceux du secteur public égyptien. Il faut qu'ils le soient en tenant compte de ce que, aujourd'hui, les salariés du secteur public ou de l'État égyptien sont contraints d'avoir un deuxième, voire un troisième emploi. Mais si l'entreprise en question estime pouvoir payer à ses salariés l'équivalent cumulé des rémunérations correspondant à ces divers emplois, voire plus, elle affirmera son dynamisme face au secteur public et sera en position favorable pour introduire de nouveaux comportements de travail. Or ces paris semblent tenables.

2 - En effet, les salaires égyptiens même, ainsi recalculés et, s'il y a lieu, majorés, semblent encore inférieurs à ceux pratiqués dans d'autres pays arabes. Un banquier interrogé estime que l'échelle des salaires, entre l'Égypte et la Péninsule arabique, serait de 1 à 7 . Elle pourrait être de 1 à 2 ou 3 , relativement à la Tunisie et de 1 à 3 ou 4 relativement au Maroc ${ }^{11}$. Un système salarial n'est jamais figé. Celui prévalant aujourd'hui en Égypte évoluera à la hausse, comme cela se produit lorsque s'affirme le développement. Il n'en reste pas moins que, actuellement, l'avantage comparatif, évalué en termes strictement quantitatifs, de ce système est reconnu par tous.

\section{Comportement et qualification de la main-d'œuvre}

On se borne à indiquer brièvement que la main-d'oeuvre égyptienne :

1. est considérée comme mieux éduquée et formée que dans bien d'autres pays en développement, en raison de l'infrastructure d'enseignement de l'Égypte, et ce, malgré la

Égypte/Monde arabe, 9 | 1992 
tendance à la baisse de l'efficience du système scolaire et universitaire, notamment dans le domaine technique.

2. est d'abord préoccupée par le souci de gagner sa subsistance et celle de sa famille, plus que travaillée par l'angoisse métaphysique, dans un contexte ou l'offre de travail, de la part des salariés, est abondante. L'influence du radicalisme musulman, bien que réelle, semble devoir être relativisée et située dans le contexte des difficultés matérielles ressenties. La motivation salariale, si la dignité de l'homme est toujours présente à l'esprit de celui qui organise et dirige le travail, apparaît donc comme un levier efficace de mise au travail de cette population.

$\mathrm{Au}$ total, et pour résumer ces perspectives de rentabilité, on reprendra le propos de l'un de nos interlocuteurs à resituer, il est vrai, en 1989 : « Le marché égyptien n'est pas un marché de haute technologie. Les activités de production intérieure sont très rentables. Les marges industrielles sont plus élevées que celles de l'Europe. Mais c'est le prix du risque, qui est grand. Les frais de personnel représentent $10 \%$ des bilans, alors qu'en France ils en représentent $45 \%$ à $50 \%$. Dans le privé, un salarié est payé cinq fois plus que dans le public. Ici, on peut vendre avec $20 \%$ à $30 \%$ de marge brute, et beaucoup le font avec $150 \%$. Ce qui est à comparer aux $2 \%$ ou $3 \%$ réalisés en Europe. » Comme on l'a déjà mentionné, investir en Égypte est une « aventure ». L'Égypte est un pays « plein de pièges ». Selon un entrepreneur ayant réussi, «c'est une entreprise sur dix qui obtient le succès désiré.»

71 Ce qui ressort, cependant, de notre enquête est que ces pièges sont évitables. Savoir qu'ils existent, prendre le temps et utiliser les moyens qu'il faut pour les éviter ou y parer, c'est se donner les meilleures chances d'accéder à un marché qui présente des caractéristiques élevées de rentabilité à moyen terme. Le paradoxe de l'entreprise moyenne est qu'elle est mieux adaptée pour en franchir les obstacles, étant plus souple dans son principe de fonctionnement.

Mais elle est aussi plus vulnérable financièrement. De plus, la conjoncture économicosociale du moment, les incertitudes politiques qui caractérisent l'anticipation de son évolution dans les cinq ans à venir, peuvent également la décourager, ou restreindre ses audaces.

Ces traits cependant se vérifient en bien d'autres endroits réputés pourtant moins dangereux que l'Égypte.

\section{NOTES}

1. Cette affirmation est d'ordre uniquement comptable et ne préjuge en rien des causes de cette situation. Le déficit de la balance commerciale est la mesure de l'excès de la dépense par rapport à l'offre. En effet, l'identité simplifiée entre les ressources et les emplois (flux) permet d'écrire, pour une économie donnée, que « Production + Importations = Consommation + Investissement + Exportations ». C'est la balance de base des biens et services réels dans un système de comptes nationaux. D'où : « P + (I $\mathrm{M})=\mathrm{C}+\mathrm{I}$ ». Le terme de droite de l'identité représente la dépense globale et celui de gauche 
représente l'offre globale intérieure, plus l'apport net en importations, tous deux nécessaires à couvrir la dépense globale. En 1987, le solde net du commerce extérieur de l'Égypte (I - M) représentait environ $10 \%$ de son produit intérieur.

2. Le présent article a été rédigé fin 1990, après dépouillement de notre enquête de mars, mais n'a pas été modifié au moment de sa publication, si ce n'est de façon marginale. En effet, le but poursuivi en 1990 n'était pas de procéder à l'analyse macro-économique de l'économie égyptienne, ce qui eût nécessité la mise à jour de nos informations et la prise en compte des réformes engagées par l'actuel gouvernement de l'Égypte, depuis janvier 1991, mais à dégager des tendances psychologiques lourdes. Cela dit, le contexte d'ensemble de cette économie est l'un des éléments à considérer, même dans le cadre d'une approche du risque égyptien, effectuée en termes de psychologie et de mentalités. Entre autres sources d'information et d'analyse de ces aspects macro-économiques, cf. par exemple Egypt, country profile, 1990-91, Annual survey of political and economical background (The Economic Intelligence Unit, London, 1990, 60 p.).

3. Cf. par exemple, de P. de CAZENOVE, Investir en Égypte, perspectives et conditions (doc. rénoté, juin 1990, Ambassade de France en RAE, 60 p., mis en vente par les soins du CFCE). Cette étude, à la différence de la nôtre, repose sur une documentation principalement statistique et réglementaire. Notre rapport d'enquête : «Les entreprises françaises et l'économie égyptienne en 1990 : le rôle des services» (doc. renoté, mars 1990, Laboratoire LAST - CLERSE, Université de Lille I, 118 p.) repose sur des entretiens non directifs (banquiers [6], industriels et commerçants [6], prestataires de services non financiers [6], membres de l'administration française au Caire [2]).

4. On note, en dehors du secteur bancaire, l'existence en Égypte de nombreuses sociétés de services dans les domaines de l'informatique ou du commerce international. Il en existe aussi dans celui du conseil juridique et fiscal destiné aux affaires, et un certain nombre dans celui de l'audit, de l'ingénierie technique, de la publicité ou du conseil en management. En revanche, les annuaires ou listings que l'on a consultés (Al-Ahrâm Directory, principalement) peu font mention de sociétés d'études en marketing basées en Égypte même. Sans doute ce moindre développement s'explique-t-il par le fait que le marché égyptien reste encore principalement offreur, et de ce fait trop étroit pour de telles sociétés. À noter, cependant, des initiatives extrêmement pointues d'exploration de tel ou tel segment du marché (celui de l'équipement en machines pour la production de textiles ou celui des produits pharmaceutiques, par exemple) mais qui ressemblent davantage à des missions longues effectuées sur place qu'à de véritables sociétés de services.

5. Le guide des postes d'expansion à l'étranger 1990 (Ed. Hera, coll. L'exportation/Les échos, p. 15-24. Cf. également notre article : «Les postes d'expansion économique français dans le monde arabe » (in France - Pays Arabes, $n^{\circ} 157$, nov. 1989, p. 19-20).

6. Cf. Investment law (law 230 for 1989) (The Middle East Library for economic services, serial $\mathrm{n}^{\circ} 30$, july 1989, 29 p.) et The executive regulation of the investment law (même éditeur que précédemment, serial $\mathrm{n}^{\circ}$ 51, december 1989, 117 p.). Pour un commentaire de la loi 230, cf. Aujourd'hui l'Égypte (hiver 1989 - 1990, p. 18-20). Sur la loi de 1974 et l'amendement de 1977, cf. SALACUSE (Jeswald W.) : Implications of Peace and openess for Égypt's legal system (1974/1980) (Reproduit dans l'annuaire égyptien des services, de l'industrie et du commerce extérieur [Al-Ahram Directory, 1988], p. 119-137), ainsi que DAVIES (Michael H.), 1984, Business law in Égypt, Kluwer, 387 p.

7. Il est possible que la nouvelle loi sur les importations (juin 1991) modifie cet aspect du risque égyptien et le réduise très sensiblement, si la mise en pratique de cette loi est conforme à l'esprit ayant présidé à son vote.

8. Cf., par exemple, les travaux de Edward T. HALL, 1971, La dimension cachée; 1979, Au delà de la culture; 1984, Le langage silencieux. Ou, dans un autre registre, l'ouvrage de Farid A. MUNA, 1980,77), The Arab executive, London, Mac-Millan, 155 p.

9. On n'a pas mentionné dans cet article le problème important du choix du partenaire ou de l'agent commercial. Compte tenu des difficultés de l'insertion dans le marché égyptien, le choix 
d'un partenaire apparaît comme une étape nécessaire pour les surmonter. Mais ce choix est luimême un risque, comme dans tous les cas d'association, de quelque nature que ce soit. Certes, ce risque peut-être surmonté et tous les dirigeants français des entreprises implantées au Caire nous ont fait l'éloge de leurs associés comme étant des "personnes remarquables ", des " perles rares ", etc. Cependant, il ressort aussi de ces entretiens que la préférence de ces dirigeants est pour la constitution de sociétés où ils seraient majoritaires. Le partenaire égyptien paraît compris comme un appui nécessaire pour la fondation de la société, mais devant rester minoritaire pour son fonctionnement. Ce qui soulève certainement d'autres problèmes que nous n'avons pas abordés dans notre enquête. On en indique cependant une première approche dans la thèse de Gérard NAULLEAU, oct. 1990, Le management des entreprises conjointes égyptiennes, analyse de la confrontation des codes socio-culturels. Thèse EHESS, Paris, $315 \mathrm{p}$.

10. Cf. P. de CAZENOVE [référence en note (3)] p. 42. Signalons, cependant, pour certains secteurs, l'existence d'études du coût salarial, plus précises que de simples comparaisons directes de taux de salaires. C'est ainsi qu'il est fait mention, dans la revue éditée par la Chambre de commerce germano-arabe (German-Égyptian Business Review, vol. 38, n³7, 1991,Special Edition on textile and Garments in Egypt, p. 39) d'une étude effectuée par Kurt Salmon Associates, sur les coûts comparés de la main-d'oeuvre en Europe et dans divers pays arabes ou autres, en 1990, pour la production de vêtements. Ce coût serait sensiblement inférieur en Égypte à celui des pays européens, mais très légèrement supérieur à celui du Maroc, de la Tunisie, de la Turquie ou de l'Inde. On peut se demander, toutefois, si ces évaluations ne sont pas remises en cause, au moins en niveau, par les évolutions actuelles des prix.

11. En mars 1990, l'évaluation du taux annuel d'inflation en Égypte était d'environ $25 \%$. Un tel taux réduit les marges brutes, mais réduit aussi les charges de l'entreprise si celle-ci s'est endettée en livres égyptiennes. Avec les réformes en cours, de nature récessionniste, ces conditions ont changé. Le taux d'inflation des prix est susceptible de ralentir très sensiblement, alors que s'élève le coût du crédit (19\% en juin 1991). L'endettement en livres égyptiennes n'est donc plus recommandé. Celui en devises étrangères continue d'être très risqué. Une entreprise étrangère s'installant en Égypte aurait donc intérêt aujourd'hui à accroître son propre apport en capital.

\section{INDEX}

Mots-clés : psychologie économique

\section{AUTEUR}

JEAN-CLAUDE DELAUNAY

CNRS, Lille I 